\title{
Early Ambulation in Patients with External Ventricular Drains: Results of a Quality Improvement Project
}

Syed Omar Shah, MD, MBA $1,2,3,4 ;$ Jacqueline Kraft, $M D^{1}$;

Nethra Ankam, MD'; Paula Bu'; Kristen Stout ${ }^{5}$; Sara Melnyk ${ }^{5}$; Fred Rincon, MD, MSC ${ }^{1,2,3,4}$; M. Kamran Athar, MD $D^{1,2,3,4}$

Departments of Neurology, ${ }^{1}$ Neurological Surgery, ${ }^{2}$ Divisions of Critical Care and Neurotrauma, ${ }^{3}$ and Cerebrovascular Diseases, ${ }^{4}$ Department of Rehabilitation Medicine, ${ }^{5}$ Thomas Jefferson University, Philadelphia, PA

Key Words

External ventricular drain, EVD, ambulation, mobilization, early ambulation

\section{Introduction}

Prolonged immobility in ICU patients can lead to muscle wasting and weakness, longer hospital stays, increased number of days in restraints and hospital acquired infections. Increasing evidence demonstrates the safety and feasibility of early mobilization in the ICU. However, there is a lack of evidence in the safety and feasibility of mobilizing patients with external ventricular drains (EVD). The purpose of this study was to determine the safety and feasibility of early mobility in this patient population.

\section{Methods}

We conducted a prospective, observational study. All patients in the study were managed with standard protocols and procedures practiced in our ICU including early mobility. Patients with an EVD that received early mobilization were awake and following commands, had a Lindegaard ratio $<3.0$ or middle cerebral artery (MCA) mean flow velocity $<120 \mathrm{~cm} / \mathrm{sec}$, a MAP> $>80 \mathrm{~mm} \mathrm{Hg}$, and an ICP consistently $<20 \mathrm{~mm}$ $\mathrm{Hg}$. Data was collected by physical therapists at the time of encounter.

\section{Results}

90 patients with a total of 185 patient encounters were recorded over a 12-month period. The average time between EVD placement and PT session was $8.3 \pm 5.5$ days. In 149 encounters (81\%), patients were at least standing or better. Patients were walking with assistance or better in 99 encounters (54\%). There were four adverse events recorded $(2.2 \%)$ during the entire study.

\section{Conclusion}

This observational study suggests that PT is feasible in patients with EVDs and can be safely tolerated. Further research in a larger patient population conducted prospectively is warranted to assess the potential benefit of early mobility in this patient population.

\section{INTRODUCTION}

Prolonged immobility in the intensive care unit (ICU) patients can lead to a myriad of complications. From loss of muscle mass and strength ${ }^{1}$ which may contribute to ICU-acquired weakness ${ }^{2,3}$ to a quality of life that remains lower than that of the general population after long-term follow-up, ${ }^{4} \mathrm{ICU}$ survivors suffer the consequences of physical inactivity during their stay. Increasing evidence demonstrates the safety and feasibility of early mobilization in the ICU. ${ }^{5}$ Specifically in the neurointensive care unit (NICU), these positive outcomes included decreased length of stay, number of patient days in restraints, number of hospital-acquired infections, and a clinically significant reduction in unit catheter-related urinary tract infection (UTI) rate. ${ }^{6}$
Increasing evidence however supports not only the benefits but also the safety of early mobilization in the NICU.6,7 Moreover, it has been shown that mechanically ventilated patients ${ }^{7}$ and patients with femoral catheters ${ }^{8}$ can be safely mobilized. There was no set protocol in place for moving these patients, nor any parameters to monitor. Upon review of the literature within physical medicine and rehabilitation, neurocritical care, and physical therapy, we found very few dedicated studies investigating mobility in patients with EVDs. Interestingly, amongst Canadian physiotherapists, those with relatively less experience were more reluctant to mobilize patients with external ventricular drains (EVD) compared to those with over 10 years of experience ${ }^{9}$. This increased reluctance has been attributed to less experienced physiotherapists being more likely to consult existing literature and rely on external sources of information such as the Guide to Physical Therapist Practice. ${ }^{9,10}$ At our institution, all patients who would be able to tolerate physical therapy are routinely evaluated and mobilized if possible, including those with EVDs. These patients are consistently mobilized by therapists with only a few years' experience, questioning the above mentioned study. Given the lack of published literature and our routine practice of mobilizing this patient population, we wanted to implement a formal protocol as a measure of a quality improvement study.

\section{METHODS}

We conducted a prospective, case series study as part of a quality improvement project. The study was approved by the Institutional Review Board of Thomas Jefferson University Hospital (IRB\# 14D.424). From June 1, 2014 until July 31, 2015, a total of 90 patients with 185 patient encounters that had EVDs occurred. All patients in the study were 
Figure 1. Safety Checklist and Data Collection Form

\section{Thomas Jefferson University Hospital}

\section{Safety and Feasibility of Early Ambulation in Patients with External Ventricular Drains: An Observational Study}

Safety Checklist: Please ask nursing staff prior to initialization of PT

1. Is the EVD closed?

2. Have the ICPs been well controlled?

3. Is the EVD secure enough for the patient to be mobilized?

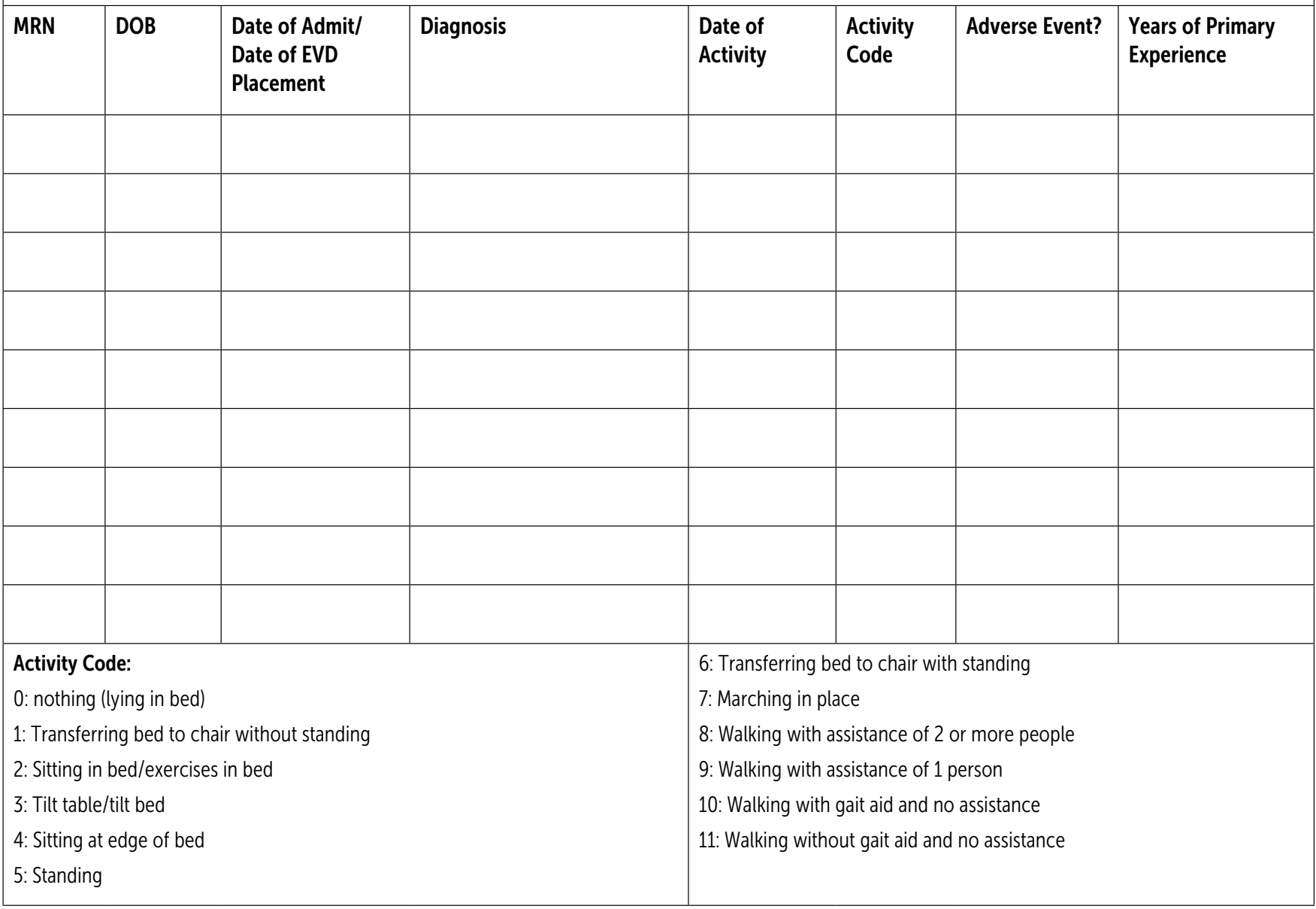

managed with standard protocols and procedures practiced in our NICU. EVDs are placed by neurological surgery residents using standard freehand pass technique with surface landmarks. It is then tunneled through the skin through a separate incision and secured by sutures. At our institution, we follow guideline based therapies for acute ischemic stroke
(AIS), intracerebral hemorrhage (ICH) and subarachnoid patients (SAH) ${ }^{11-13}$ All patients are initially on bedrest on admission day, and then evaluated for physical therapy the next day. To be evaluated for physical therapy, the patient had to be able to ambulate. Our protocol for $\mathrm{SAH}$ patients who were deemed eligible for physical therapy were similar to a recently published study by Olkowski et al. ${ }^{14}$ Patients with $\mathrm{SAH}$ were deemed eligible for early mobilization if they were awake and following commands, had a Lindegaard ratio $<3.0$ or middle cerebral artery(MCA) mean flow velocity $<120 \mathrm{~cm} / \mathrm{sec}$, a MAP>80 $\mathrm{mm} \mathrm{Hg}$, and an intracranial pressure (ICP) consistently $<20 \mathrm{~mm} \mathrm{Hg}$. For patients with $\mathrm{ICH}$, a 
Table 1. Age, admitting diagnosis and types of therapies tolerated. SD - Standard Deviation.

\begin{tabular}{|c|c|c|}
\hline & & Percent of Total \\
\hline Age, X (SD) & $56(14)$ & \\
\hline Female & 49 & 54 \\
\hline Male & 42 & 46 \\
\hline Admitting GCS, median (IQR) & $11(8-15)$ & \\
\hline GCS on Ambulation, median (IQR) & $15(14-15)$ & \\
\hline \multicolumn{3}{|l|}{ Admitting Diagnoses } \\
\hline Subarachnoid Hemorrhage & 45 & 50 \\
\hline Hunt Hess Grade, median (IQR) & $3(3-4)$ & \\
\hline Modified Fisher, median (IQR) & $4(3-4)$ & \\
\hline Intracerebral Hemorrhage & 23 & 26 \\
\hline ICH Score, median (IQR) & $2(1-2)$ & \\
\hline Tumor & 12 & 13 \\
\hline Ischemic Strokes & 3 & 3 \\
\hline NIHSS on Admission, median (IQR) & $12(9-21)$ & \\
\hline Chronic Hydrocephalus & 6 & 7 \\
\hline Trauma & 1 & 1 \\
\hline Total Patients & 90 & \\
\hline
\end{tabular}

then entered in our study. Patients were excluded if they were delirious using the CAM-ICU score. Intubated patients were also excluded as our patient population is exclusively neurologically injured patients. Patients in our unit who are mechanically ventilated, for the most part have a poor Glasgow Coma Scale (GCS) rather than have a primary lung injury. For this study, we thought it prudent to evaluate patients who were not mechanically ventilated first and then add this patient population to further projects.

The protocol implemented included an initial assessment by the physician on service to determine readiness to participate in physical therapy. If deemed appropriate, physical therapy (PT) and occupational therapy (OT) consult were requested. Therapy was then initiated after the nurse clamped the EVD and if the ICPs were less than $20 \mathrm{~mm} \mathrm{Hg}$ for greater than $30 \mathrm{~min}$.

Physical therapists completed a sheet as they performed their assessments. The date of admission and EVD placement, type of activity, years of experience of the primary therapist, and the number of people mobilized in a day were recorded. Activity codes utilized were based on forms obtained from Dale Needham at Johns Hopkins University with his permission. Physical therapists were also instructed to record changes in vital signs, arrhythmia, changes in ICPs, and/ or dislodging lines. All adverse events were recorded. If an adverse event occurred during a therapy session, the session was immediately stopped and the attending physician was notified.

A preplanned interim analysis was conducted to review adverse events after a 6-month data collection period. Investigators discussed the adverse events that occurred and then created a questionnaire to be filled out by the therapy team before each mobilization session in order to prevent further adverse events. The patients must have an EVD that was clamped, ICPs that are well controlled and an EVD that is well secured. Protocol was adjusted so that the physical therapists would have to answer yes to all questions before any initiation of therapy. The resulting questionnaire is shown in Figure 1.

follow-up computed tomography (CT) scan is obtained 24 hours after the initial bleed at our institution. This is done to determine stability of the hemorrhage in order to begin subcutaneous heparin for deep vein thrombosis prophylaxis. If the repeat $C T$ scan was stable and ICPs were consistently $<20 \mathrm{~mm} \mathrm{Hg}$, the patient was also then deemed eligible for early mobilization. Patients with AIS, traumatic brain injury, tumors or chronic hydrocephalus were deemed appropriate if the ICPs were consistently $<20 \mathrm{~mm} \mathrm{Hg}$ All patients that met these criteria were

\footnotetext{
tionnaire is shown in Figure 1.
}




\section{RESULTS}

Ninety patients with a total of 185 patient encounters were recorded over a 12-month period. Table 1 summarizes the admitting diagnoses and types of therapy tolerated by the patients. The mean age was $56 \pm 14$ years old. The average time between EVD placement and PT session for patients with $\mathrm{SAH}$ was $7.7 \pm 4.5$ days. $\mathrm{ICH}$ and tumor patients were seen on day $6.6 \pm 3.1$ days and $8.1 \pm 5.8$ days. Subarachnoid hemorrhage patients had a median Hunt Hess Grade of 3 and a modified Fisher grading scale of 4 . Intracerebral hemorrhage patients had an ICH score of 2 .

A priori, we determined adverse events to be any event that caused an abrupt termination of therapy. Four adverse events were recorded (2.2\%) during the entire study. Prior to the interim analysis, there were two adverse events in 132 patient encounters. The first event was an increase in ICP after patient went from supine to sitting position. The second event was dislodgement of the EVD while walking the patient. At the interim analysis both cases were reviewed in detail and an adjustment to the protocol was made as discussed above. Post interim analysis, there were two events. The first event was patient emesis after rolling the patient in bed. The second event was an increase in ICP. No changes in neurological exam were recorded during any of the four adverse events. Although not directly part of this study, we did not see any secondary complications of SAH such as vasospasm or $\mathrm{DCl}$ as a consequence of mobilizing this patient population.

To determine the feasibility of mobilizing these patients, we determined what type of therapy was tolerated by the patients. In 149 encounters (81\%), patients were at least standing or better. Patients were walking with assistance or better in 99 encounters (54\%). Only $3.8 \%$ of patients had passive range of motion in the bed.

\section{DISCUSSION}

After implementation of the new safety checklist, there were no further EVD dislodgements. We attribute this to the question in the checklist that questions whether the EVD is secure enough to have the patient manipulated. As with any drain or line, the longer it stays in the patient the greater the chance for dislodgement. During the preplanned interim analysis, it was discovered that the dislodged EVD was in fact not securely in place before the PT session.

We routinely drain EVDs when ICPs are greater than $20 \mathrm{~mm} \mathrm{Hg}$. Both ICP events were increases in ICP no higher than $22 \mathrm{~mm} \mathrm{Hg}$. After prompt cerebrospinal fluid (CSF) drainage, the ICPs returned to normal. Although the checklist does ask whether the ICPs have been controlled, ICPs in general can fluctuate at any given time. Moreover, our patients only have limited mobility until they are seen by the physical therapy team. This encounter may be the first instance in which the patient's ICP compliance is truly tested.

Our average day until PT initiation was longer than what was recently reported by Olkowski, 2.3 days versus 7.7 for $\mathrm{SAH}$ patients. ${ }^{14}$ We attribute this to our patient population having higher grade $\mathrm{SAH}$. Also all of our patients have EVD placed, while Olkowski included patients without EVD as well. Patients that require EVD placement require longer time for their mental status to improve.

Increasing evidence supports not only the benefits but also the safety of early mobilization in the NICU. In a study investigating early mobilization in aneurysmal subarachnoid hemorrhage patients, $32 \%$ of patients had external ventricular drains placed. In this group, only $5.9 \%$ of early mobilization program sessions had an adverse event and such a program was concluded to be safe and feasible ${ }^{14}$. Adverse events were a MAP $<70 \mathrm{~mm} \mathrm{Hg}$ or $>120 \mathrm{~mm} \mathrm{Hg}$, or heart rate $>130$ bpm. Very early mobilization of stroke patients within 24 hours of stroke onset resulted in patients returning to independent walking sooner and remaining more independent in motor function at 12 months ${ }^{15}$. Bimmouille et al. demonstrated that patients with normal and elevated ICP could perform most exercises with physical therapy without changes in ICP, with the exclusion of hip flexion ${ }^{16}$. Titsworth et al ${ }^{6}$ reported there was no significant difference in the total number of falls, fall rate per 1000 patient days, or critical line pull rate before and after a comprehensive mobility initiative.
While clear recommendations cannot be inferred from this study, our study does show that early mobilization in patients with EVDs can be safely performed. Only $2.2 \%$ of encounters had significant events and none of these events caused significant harm to the patient. Although a dislodgement of an EVD may cause immediate harm, our patient was able to tolerate the removal without any change in neurological exam.

Our study has several limitations. This is a not a randomized trial and thus we can only report association and not causation. Secondly, there is bias to our data collection as we only included patients that had PT orders placed in our electronic medical record system. There may have been potential participants who were missed. Moreover, patients in whom the primary team decided not to order PT consult due to these patients being deemed unable to tolerate PT, need to be considered. Lastly, this study is done at a single center institution that has access to a team of physical therapists, occupational therapists and physiatrists which may not be feasible for all hospitals.

The results of this quality improvement study allowed us to implement a protocol for early mobilization in patients with EVDs and make adjustments to the protocol as needed. With the institution of a standard protocol for mobilizing these patients, along with a safety checklist, we had few adverse events. With this preliminary data, a randomized controlled trial that examines the safety and feasibility is warranted in this patient population. A larger randomized controlled trial would also be better to evaluate if early ambulation decreases delirium, length of stay, or improves outcomes in patients with EVDs.

\section{REFERENCES}

1. Ferrando AA, Lane HW, Stuart CA, Davis-Street J, Wolfe RR. Prolonged bed rest decreases skeletal muscle and whole body protein synthesis. Am J Physiol. 1996;270:E627-633

2. Fan E. Critical illness neuromyopathy and the role of physical therapy and rehabilitation in critically ill patients. Respir Care. 2012;57:933944; discussion 944-936 
3. Kortebein P, Ferrando A, Lombeida J, Wolfe $\mathrm{R}$, Evans WJ. Effect of 10 days of bed rest on skeletal muscle in healthy older adults. JAMA 2007:297:1772-1774

4. Dowdy DW, Eid MP, Sedrakyan A, MendezTellez PA, Pronovost PJ, Herridge MS, et al. Quality of life in adult survivors of critical illness: A systematic review of the literature. Intensive care medicine. 2005;31:611-620

5. Morris PE, Herridge MS. Early intensive care unit mobility: Future directions. Crit Care Clin 2007:23:97-110

6. Titsworth WL, Hester J, Correia T, Reed R, Guin P, Archibald L, et al. The effect of increased mobility on morbidity in the neurointensive care unit. Journal of neurosurgery. 2012;116:1379-1388

7. Schweickert WD, Pohlman MC, Pohlman AS, Nigos C, Pawlik AJ, Esbrook CL, et al. Early physical and occupational therapy in mechanically ventilated, critically ill patients: A randomised controlled trial. Lancet. $2009 \cdot 373 \cdot 1874-1882$

8. Perme C, Nalty T, Winkelman C, Kenji Nawa R, Masud F. Safety and efficacy of mobility interventions in patients with femoral catheters in the icu: A prospective observational study. Cardiopulm Phys Ther J. 2013;24:12-17
9. Hale C, Wong K, Pennings A, Rnic A, Tobali B, Hawke C, et al. Practice patterns of canadian physiotherapists mobilizing patients with external ventricular drains. Physiother Can.

10. Wainwright SF, Shepard KF, Harman LB, Stephens J. Factors that influence the clinical decision making of novice and experienced physical therapists. Phys Ther. 2011;91:87-101

11. Connolly ES, Jr., Rabinstein AA, Carhuapoma JR, Derdeyn CP, Dion J, Higashida RT, et al. Guidelines for the management of aneurysmal subarachnoid hemorrhage: A guideline for healthcare professionals from the american heart association/american stroke association. Stroke; a journal of cerebra circulation. 2012;43:1711-1737

12. Hemphill JC, 3rd, Greenberg SM, Anderson CS, Becker K, Bendok BR, Cushman M, et al. Guidelines for the management of spontaneous intracerebral hemorrhage: A guideline for healthcare professionals from the american heart association/american stroke association. Stroke; a journal of cerebral circulation. 2015:46:2032-2060

13. Jauch EC, Saver JL, Adams HP, Jr., Bruno A, Connors JJ, Demaerschalk BM, et al. Guidelines for the early management of patients with acute ischemic stroke: A guideline for healthcare professionals from the american heart association/american stroke association. Stroke; a journal of cerebral circulation. 2013;44:870-947
14. Olkowski BF, Devine MA, Slotnick LE, Veznedaroglu E, Liebman KM, Arcaro ML, et al. Safety and feasibility of an early mobilization program for patients with aneurysmal subarachnoid hemorrhage. Phys Ther. 2013; $93: 208-215$

15. Cumming TB, Thrift AG, Collier JM, Churilov L, Dewey HM, Donnan GA, et al. Very early mobilization after stroke fast-tracks return to walking: Further results from the phase ii avert randomized controlled trial. Stroke; a journal of cerebral circulation. 2011;42:153-158

16. Brimioulle S, Moraine JJ, Norrenberg D, Kahn RJ. Effects of positioning and exercise on intracranial pressure in a neurosurgical intensive care unit. Phys Ther. 1997;77:1682-1689

\section{Corresponding Author}

\section{Syed Omar Shah, MD MBA}

Assistant Professor

Department of Neurological Surgery

Thomas Jefferson University Hospital

909 Walnut St, 3rd Floor

Philadelphia, PA 19107

\section{P: $215-503-5550$}

F. 215-503-3301

E: syed.o.shahajefferson.edu

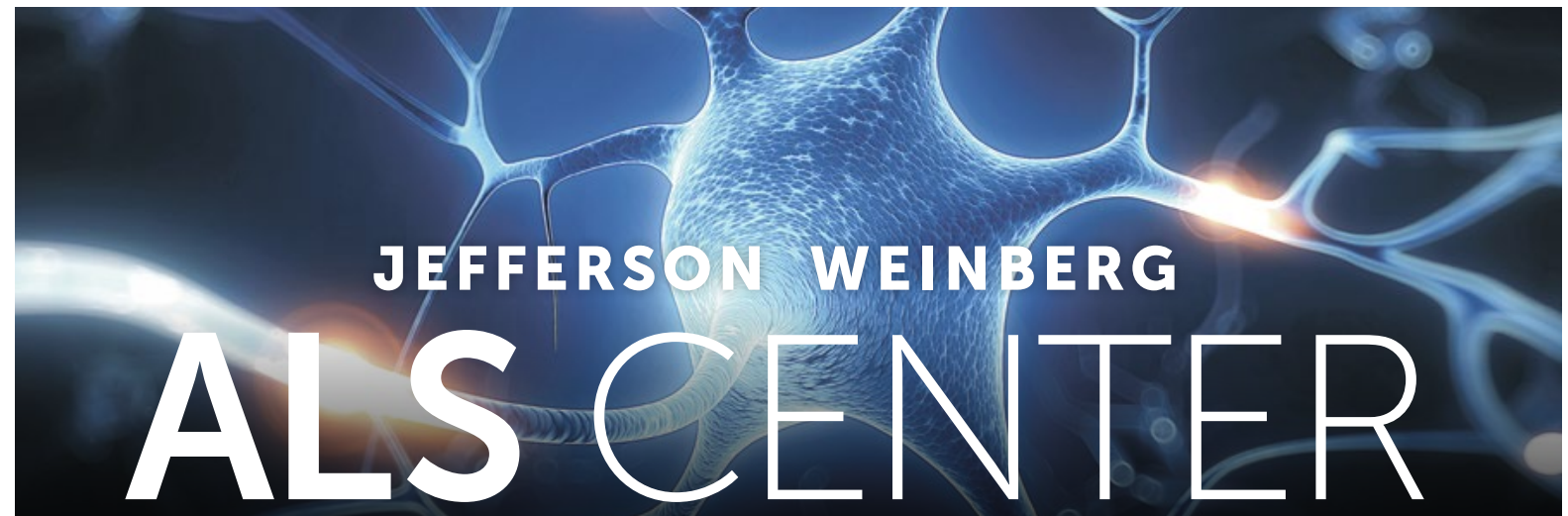

The Vickie and Jack Farber Institute for Neuroscience at Jefferson, in partnership with The ALS Association, Greater Philadelphia Chapter, is pleased to announce the creation of the Jefferson Weinberg ALS Center, which builds upon Jefferson's leadership in basic ALS research by adding a new multidisciplinary ALS clinic.

Jefferson.edu/ALS | 909 Walnut Street, Philadelphia, PA

Q Vickie and Jack Farber Institute for Neuroscience. at Jefferson 